\title{
Características de reporte e condução de revisões sistemáticas que avaliaram a longevidade de restaurações de resina composta em dentes posteriores
}

\author{
Reporting and conduction characteristics of systematic reviews that \\ evaluated the longevity of composite resin restorations in posterior teeth
}

Alana Geisiane da Rosa Aquino*

Camila Raubach Dias*

Fernanda Vieira Almeida*

Gabriel Nunes Valduga*

Gabriela Cardoso Vida*

Rafael Sarkis-Onofre ${ }^{* *}$

\section{Resumo}

Muitas revisões avaliando a longevidade de restaurações de resina composta em dentes posteriores vem sendo publicadas e o melhor entendimento dessas revisões é importante. Objetivo: o objetivo do presente estudo foi investigar as características de condução e de reporte das revisões sistemáticas que avaliaram a longevidade de restaurações de resina composta em dentes posteriores. Materiais e método: foi realizada uma busca no MedLine (via Pubmed) com objetivo de identificar revisões que analisaram apenas a longevidade de restaurações de resina composta e revisões que compararam restaurações de resina composta com outros materiais em dentes posteriores. Os artigos foram selecionados de forma independente por dois revisores baseados inicialmente na leitura do título/resumo e depois na leitura do artigo completo. Características dos periódicos em que os artigos foram publicados, do reporte e da condução das revisões foram extraídas. Tabelas foram criadas para sumarizar os dados. Resultados: no total, 11 artigos foram incluídos pela síntese qualitativa. A maioria das revisões utilizou meta-análise $(63,6 \%)$ e a maior parte $(90,9 \%)$ mencionou os termos revisão sistemática/meta-análise no título ou no resumo. Sete revisões $(63,6 \%)$ não mencionaram a utilização de algum guia de reporte. Grande parte das revisões $(54,5 \%)$ não incluiu apenas ensaios clínicos randomizados e oito estudos $(72,2 \%)$ avaliaram o risco de viés e apresentaram o diagrama de fluxo da seleção dos estudos. Conclusão: pode-se concluir que muitas revisões sobre a longevidade de restaurações vêm sendo publicadas na literatura odontológica, sendo muitas delas conduzidas e reportadas de maneira diferente, $o$ que pode impactar diretamente na qualidade do estudo e na aplicabilidade dos resultados.

Palavras-chave: Longevidade. Restauração dentária permanente. Resinas compostas. Revisão.

\section{Introdução}

As restaurações de resina composta são amplamente utilizadas em dentes posteriores com a finalidade de restabelecer forma e função a dentes acometidos por cárie, atrição, fraturas e outros fatores. Dessa forma, representa uma das atividades mais desenvolvidas nos consultórios odontológicos devido à rapidez do tratamento, ao baixo custo e excelente resultado estético ${ }^{1,2}$.

A longevidade de restaurações está relacionada com o tempo que essa restauração permanece em boca de forma aceitável. Conhecer e avaliar a longevidade e os fatores envolvidos é importante, uma vez que a troca repetitiva de material desgasta o dente e pode acarretar perda de tecido dentário sa- 
dio. Alguns fatores podem influenciar a longevidade de restaurações de dentes posteriores incluindo o diagnóstico correto do dentista, a técnica restauradora utilizada, os hábitos alimentares e de higiene oral do paciente, a frequência com que se troca de dentista, o tipo e a localização do dente, o risco de cáries, de estresse oclusal - como bruxismo - e desordem temporomandibular ${ }^{3}$.

Revisões sistemáticas (RS) são estudos que tentam coletar toda evidência que se enquadra em um critério de inclusão pré-estabelecido para responder uma questão específica de pesquisa ${ }^{4}$. Esse é um tipo de estudo que se encontra no topo da pirâmide de evidência e possui grande importância devido ao seu método explícito e sistemático para identificar, selecionar e avaliar a qualidade de evidências, sendo produzido por uma metodologia confiável e de alto rigor. Atualmente, muitas RS vêm sendo publicadas na literatura incluindo estudos repetidos o que poderia acarretar em desperdício de dinheiro e tempo pelos pesquisadores ${ }^{5}$. Muitas revisões avaliando a longevidade de restaurações de resina composta em dentes posteriores vem sendo publicadas e o melhor entendimento sobre como essas revisões vem sendo conduzidas e reportadas é importante.

Assim, o objetivo do estudo foi investigar as características epidemiológicas e de reporte das RS que avaliaram a longevidade de restaurações de resina composta em dentes posteriores.

\section{Materiais e método}

\section{Critérios de elegibilidade}

Os critérios de elegibilidade dos estudos se basearam em dois pontos principais: 1) ser uma revisão sistemática e 2) avaliar a longevidade de restaurações de resina composta em dentes posteriores. Foram incluídas RS baseadas na definição do PRISMA- $\mathrm{P}^{6}$ que define que uma revisão sistemática deve apresentar um método de identificação dos estudos (ex.: estratégia de busca), um método de seleção dos estudos (ex.: critério de elegibilidade e processo de seleção) e descrever um método de síntese ou outro tipo de sumário.

Quanto à avaliação da longevidade de restaurações de resina composta em dentes posteriores foram incluídas tanto revisões que analisaram apenas a longevidade da resina composta quanto às revisões que compararam resina com outros materiais. As resinas avaliadas nas revisões poderiam ser tanto diretas como indiretas. Foram excluídos qualquer tipo de revisão que não se enquadrasse no critério acima citado, que avaliaram fatores relacionados as resinas compostas (ex: adesivos) ou que não fossem revisões originais, como resumos de revisões anteriormente publicadas.

\section{Busca}

A busca inicial foi realizada no Pubmed, sem restrição de linguagem e com a utilização dos termos Mesh relacionados ao tema e um filtro específico para $\mathrm{RS}^{7}$. A estratégia de busca apresenta-se no Quadro 1. Não houve restrição de tempo e a última busca foi realizada em setembro de 2016.

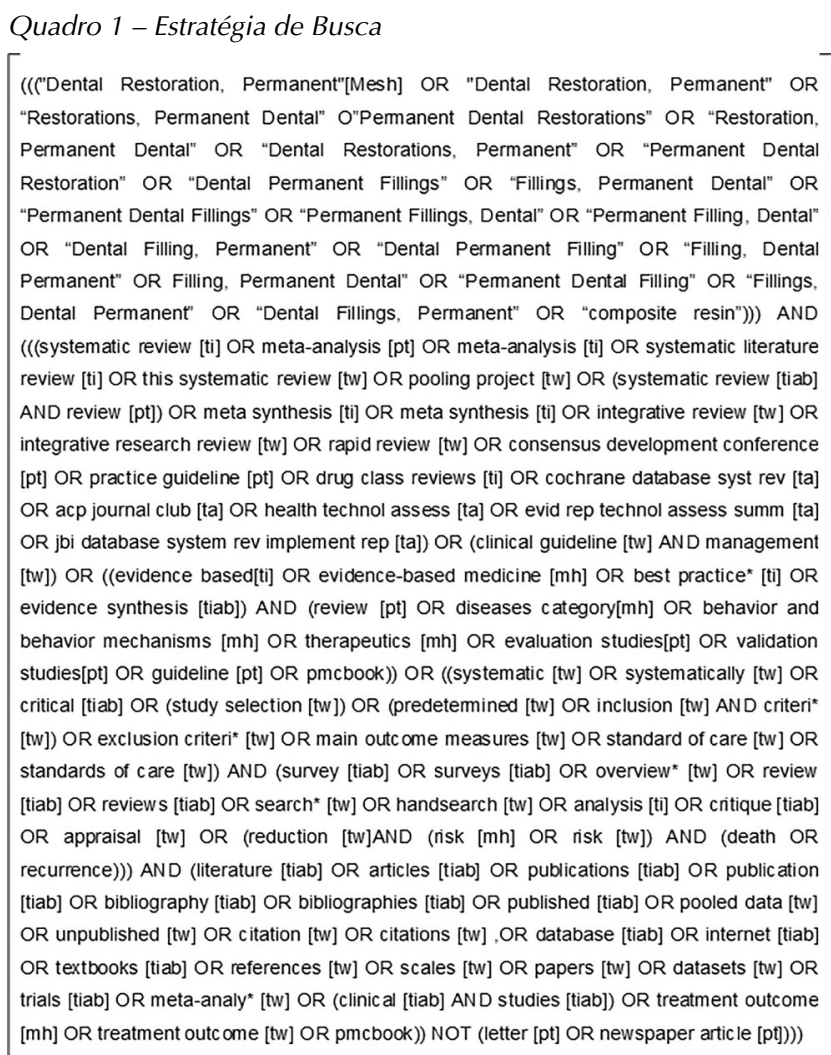
standards of care [tw]) AND (survey [tiab] OR surveys [tiab] OR overview* [tw] OR review [tiab] OR reviews [tiab] OR search* [tw] OR handsearch [tw] OR analysis [t] OR critique [tiab] $O R$ appraisal [tw] $O R$ (reduction [tw]AND (risk [mh] OR risk [tw]) AND (death $O R$ recurrence))) AND (literature [tiab] OR articles [tiab] OR publications [tiab] OR publication [tiab] OR bibliography [tiab] OR bibliographies [tiab] OR published [tiab] OR pooled data [tw] OR unpublished [tw] OR citation [tw] OR citations [tw] , OR database [tiab] OR internet [tiab] OR textbooks [tiab] OR references [tw] OR scales [tw] OR papers [tw] OR datasets [tw] OR trials [tiab] OR meta-analy* [tw] OR (clinical [tiab] AND studies [tiab]) OR treatment outcome [mh] OR treatment outc ome [tw] OR pmcbook)) NOT (letter [pt] OR newspaper article [pt])))

\section{Seleção dos estudos}

Após a busca, a seleção dos estudos foi realizada por duas pessoas de maneira independente. Inicialmente, foi feita a leitura dos títulos e resumos de cada artigo baseados nos critérios de inclusão acima citados e classificando os artigos em incluir, excluir e dúvida. Os artigos classificados como dúvida e incluir foram escolhidos para leitura completa também de forma independente e foram aplicados novamente os critérios de elegibilidade. Após a leitura completa dos artigos, em caso de dúvida, uma reunião de consenso entre os revisores era realizada e caso a dúvida permanecesse um terceiro revisor era consultado.

\section{Extração e análise dos dados}

A extração dos dados dos estudos incluídos no trabalho foi feita através da criação de uma tabela padronizada do Excel com as seguintes informações: autor, ano de publicação, periódico, número de autores, país do autor correspondente, número de 
estudos incluídos, se uma meta-análise foi realizada ( $\operatorname{sim}$ ou não), se os termos revisão sistemática e/ ou a meta-análise foram mencionados no título ou no resumo (sim ou não), se foi usado um guia de reporte (sim ou não), qual o guia de reporte foi usado, o número de bases pesquisadas, se incluiu apenas ensaios clínicos randomizados, se avaliou o risco de viés, se apresentou diagrama de fluxo e a conclusão das revisões. Inicialmente foi realizada de consenso entre todos os pesquisadores envolvidos na pesquisa. A extração de todos os dados foi realizada em duplicata por dois grupos independentes. A partir dos dados obtidos foram criadas tabelas a fim de sumarizar os dados.

\section{Resultados}

A Figura 1 mostra o diagrama de fluxo com os resultados da seleção dos estudos. A pesquisa inicial resultou em 48 artigos dos quais 33 foram excluídos baseados na análise do título e resumo. Foram avaliados então, 15 artigos na íntegra para elegibilidade, e desses, 4 foram excluídos com as seguintes razões: 1) Não apresentaram método explícito; 2) Resumo de uma revisão; e 3) Objetivo principal não era avaliar a longevidade das restaurações. Portanto, 11 artigos foram incluídos pela síntese qualitati$\mathrm{va}^{8-18} \mathrm{e}$, desses, extraídos dados relevantes ao tema.

A Tabela 1 mostra as características dos estudos incluídos e as comparações nas revisões. Dos estudos incluídos quatro comparam amálgama com resina composta em dentes posteriores sendo que três destes favorecem o amálgama e o outro relata que a longevidade é semelhante. Outros quatro estudos comparam restaurações de resina composta do tipo Inlay/Onlay com cerâmica e todos demonstraram que os dois materiais apresentam longevidade semelhante e altas taxas de longevidade. A comparação da resina composta com o ionômero de vidro apareceu em somente um dos estudos incluídos e concluiu que a taxa de falha entre esses materiais é equivalente. Das revisões que avaliaram somente a resina composta em dentes posteriores uma cita que a taxa de falha é de $1,8 \%$ em cinco anos e de $2,4 \%$ em 10 anos, e a outra aponta elevado desempenho da resina composta.

A Tabela 2 apresenta as características dos periódicos em que foram publicados tais estudos. Ao todo, esses estudos foram publicados em sete periódicos distintos, sendo que, desses sete jornais, apenas dois $(28,6 \%)$ apresentam mais de duas RS publicadas. Desses sete periódicos, três deles $(42,9 \%)$ possuem fator de impacto maior que 2 . Além disso, a maioria dos estudos $(72,8 \%)$ tem publicação a partir do ano de 2014 e a Europa representa o continente com a maior parte $(72,7 \%)$ de autores correspondentes.
A Tabela 3 expõe características de como as RS foram realizadas e reportadas. O número mediano de autores foi 4 (AIQ 3-6) e de estudos incluídos foi 12 (AIQ 6,5-16). A maioria das revisões utilizou meta-análise $(63,6 \%)$ como forma de sumarizar os resultados e a maior parte $(90,9 \%)$ mencionou os termos revisão sistemática/meta-análise no título ou no resumo. Sete revisões $(63,6 \%)$ não mencionaram a utilização de algum guia de reporte e quatro delas $(36,4 \%)$ utilizaram o guia de reporte PRISMA. Grande parte das revisões $(54,5 \%)$ não incluiu apenas os ensaios clínicos randomizados e oito estudos $(72,2 \%)$ avaliaram o risco de viés e apresentaram o diagrama de fluxo da seleção dos estudos.

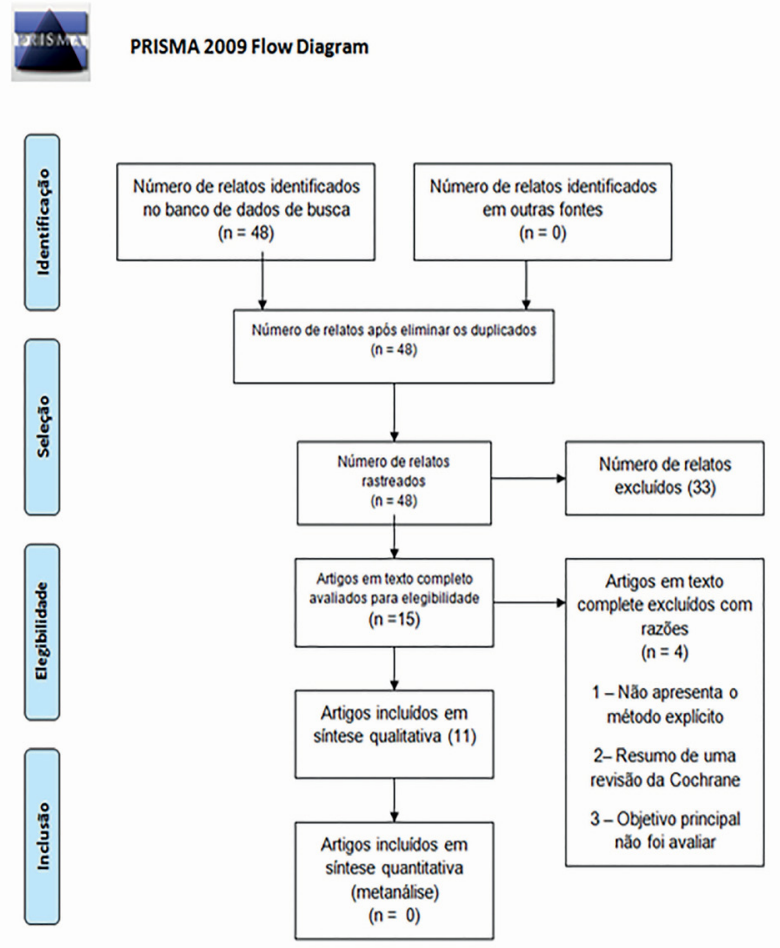

Figura 1 - Diagrama de fluxo da seleção dos estudo 
Tabela 1 - Características das revisões sistemáticas das que avaliaram a longevidade de resina composta em dentes posteriores considerando o ano de publicação, nome do periódico que foi publicado o artigo, comparação testada e conclusão do estudo

\begin{tabular}{|c|c|c|c|c|}
\hline Autor & $\begin{array}{c}\text { Ano de } \\
\text { publicação }\end{array}$ & Periódico & Comparação & Conclusão \\
\hline Antony et al. & 2008 & $\begin{array}{l}\text { GMS Health Technology } \\
\text { Assessment }\end{array}$ & Amálgama x Resina Direta & Favorece Amálgama \\
\hline Heintze et al. & 2012 & Journal of Adhesive Dentistry & $\begin{array}{l}\text { Restaurações classe II de resi- } \\
\text { na x amálgama }\end{array}$ & Longevidade semelhante \\
\hline Chabouis et al. & 2013 & Journal of Dentistry & $\begin{array}{l}\text { Inlays e Onlays de resina } x \\
\text { cerâmica }\end{array}$ & Desempenho semelhante \\
\hline Opdam et al. & 2014 & Journal of Dental Research & $\begin{array}{l}\text { Restaurações diretas de resina } \\
\text { composta }\end{array}$ & $\begin{array}{l}\text { Taxas de falha anuais de } \\
1,8 \% \text { em } 5 \text { anos e } 2,4 \% \text { após } \\
10 \text { anos de serviço. }\end{array}$ \\
\hline Rasines Alcaraz et al. & 2014 & $\begin{array}{l}\text { Cochrane Database of Systematic } \\
\text { Reviews }\end{array}$ & Amálgama x Resina Direta & Favorece Amálgama \\
\hline Grivas et al. & 2014 & $\begin{array}{l}\text { European Journal of Prosthodon- } \\
\text { tics and Restorative Dentistry }\end{array}$ & $\begin{array}{l}\text { Inlays e Onlays de resina x ce- } \\
\text { râmica x ouro x resina direta }\end{array}$ & $\begin{array}{l}\text { Inlays de resina composta pa- } \\
\text { recem ser um método efetivo }\end{array}$ \\
\hline Moraschini et al. & 2015 & Journal of Dentistry & Amálgama x Resina Direta & Favorece Amálgama \\
\hline Mickenautsch et al. & 2015 & The Open Dentistry Journal & $\begin{array}{l}\text { Cimento de iônomero de vi- } \\
\text { dro } x \text { resina composta }\end{array}$ & Taxa de falha semelhante \\
\hline Ástvaldsdóttir et al. & 2015 & Journal of Dentistry & $\begin{array}{l}\text { Restaurações de resina com- } \\
\text { posta }\end{array}$ & Alta taxa de sobrevida \\
\hline Morimoto et al. & 2016 & Journal of Dental Research & $\begin{array}{l}\text { Inlays, Onlays e Overlays de } \\
\text { resina x cerâmica }\end{array}$ & $\begin{array}{l}\text { Altas taxas de sobrevivência } \\
\text { para todos materiais }\end{array}$ \\
\hline Angeletak et al. & 2016 & Journal of Dentistry & $\begin{array}{l}\text { Inlays e Onlays de resina dire- } \\
\text { ta } x \text { indireta }\end{array}$ & Longevidade semelhante \\
\hline
\end{tabular}

Tabela 2 - Característica dos Periódicos que publicaram os estudos incluídos

\begin{tabular}{|c|c|c|}
\hline Características & Categoria & Número (\%) \\
\hline $\begin{array}{l}\text { Número total de } \\
\text { periódicos }\end{array}$ & - & 7 \\
\hline \multirow[t]{4}{*}{$\begin{array}{l}\text { Número de RS por } \\
\text { periódico }\end{array}$} & 1 & $5(71,4)$ \\
\hline & 2 & $0(0)$ \\
\hline & 3 & $1(14,3)$ \\
\hline & 4 & $1(14,3)$ \\
\hline \multirow[t]{3}{*}{$\begin{array}{l}\text { Fator de impacto do } \\
\text { periódico (2015) }\end{array}$} & Sem fator de impacto & $3(42,9)$ \\
\hline & $0,0-2,0$ & $1(14,3)$ \\
\hline & $>2,0$ & $3(42,9)$ \\
\hline \multirow[t]{6}{*}{ Ano de publicação } & 2008 & $1(9,1)$ \\
\hline & 2012 & $1(9,1)$ \\
\hline & 2013 & $1(9,1)$ \\
\hline & 2014 & $3(27,3)$ \\
\hline & 2015 & $3(27,3)$ \\
\hline & 2016 & $2(18,2)$ \\
\hline \multirow[t]{3}{*}{$\begin{array}{l}\text { País do autor } \\
\text { correspondente }\end{array}$} & África & $1(9,1)$ \\
\hline & América & $2(18,2)$ \\
\hline & Europa & $8(72,7)$ \\
\hline
\end{tabular}

Tabela 3 -Características metodológicas das revisões sistemáticas das que avaliaram a longevidade de resina composta em dentes posteriores

\begin{tabular}{l} 
Características \\
\hline Número de autores \\
Número de estudos incluídos \\
Comparação testada \\
Amálgama x Resina Direta \\
Inlay, Onlay e overlays \\
Apenas resina composta \\
CIV x Resina Composta \\
Metanálise realizada \\
Sim \\
Não
\end{tabular}

Categoria

\begin{tabular}{|c|c} 
Categoria \\
\hline
\end{tabular}

$12(6,5-16)$

$4(36,4)$

$4(36,4)$

$2(18,2)$

$1(9,1)$

$7(63,6)$

$4(36,4)$

Revisão sistemática/metanálise mencionada no título ou resumo

Sim

$10(90,9)$

Não

$1(9,1)$

Uso de guia de reporte

Sim

$4(36,4)$

$7(63,6)$

Não

$3(2,5-3,5)$

Número de bases pesquisadas

Incluiu apenas ECRs

$\operatorname{Sim}$

$5(45,5)$

Não

$6(54,5)$

Avaliou o risco de viés

Sim

$8(72,7)$

Não

$3(27,3)$

Apresenta o diagrama de fluxo

Sim

$8(72,7)$

Não

$3(27,3)$

Dados expresso como número (\%) ou mediana (AIQ). 


\section{Discussão}

Esse estudo é o primeiro a identificar RS que avaliaram a longevidade de restaurações de resina composta em dentes posteriores e avaliar as características de como elas foram conduzidas e reportadas. O estudo se torna importante, pois RS apresentam o maior nível de evidência visto que estão localizadas no topo da pirâmide de evidência e as restaurações de resina compostas representam uma das atividades mais desenvolvidas nos consultórios odontológicos ${ }^{1,2}$.

Foram localizadas, no total, 11 revisões sobre 0 tema com diferenças significativas na condução e no reporte. A condução e o reporte das revisões apresentaram aspectos positivos e negativos. De maneira negativa algumas diferenças acabam ocasionando problemas na qualidade da revisão como, por exemplo: $63,4 \%$ das revisões selecionadas não utilizarem nenhum guia de reporte para basear a escrita do artigo, $27,3 \%$ não avaliar o risco de viés dos estudos incluídos e 27,3\% não apresentar o diagrama de fluxo da seleção dos artigos. De maneira positiva o principal aspecto a ser destacados é que $90,9 \%$ dos estudos incluídos mencionarem o termo "Revisão sistemática/metanálise" no título ou resumo que é uma das recomendações do PRISMA Statement ${ }^{4}$.

Esses achados são importantes visto que problemas na condução podem acarretar em resultados imprecisos e problemas no reporte dificultam os leitores de determinar a validade dos métodos utilizados. Essa variação das características das revisões encontrada nesse estudo estão de acordo com achados recentes da literatura que mostraram uma variação muito grande na condução e no reporte de RS publicadas na literatura biomédica ${ }^{19}$. No entanto, é importante ressaltar que mesmo os estudos apresentando essas diferenças no geral eles apresentaram resultados consistentes.

Esse estudo identificou que a maioria das revisões nessa temática foram publicadas a partir de 2013. Estudos recentes vêm demonstrando um aumento do número de RS publicadas nos últimos anos com estimativa de que 22 revisões são publicadas diariamente no MEDLINE ${ }^{19-21}$. Algumas explicações podem estar relacionadas ao grande número de revisões publicadas: algumas agências de fomento requerem que pesquisadores ao submeterem projetos referenciem o projeto com uma RS ou realize uma RS se ainda não existir, países como China vem desenvolvendo uma cultura de pesquisa com grande ênfase na produção desse tipo de estudo e o desenvolvimento de programas de livre acesso de estatística que realizam meta-análise. Além disso, o fato de alguns países incentivarem a produção em massa de artigos (ex: maiores recursos de pesquisas para quem publica maior número de artigos) e a promoção na carreira acadêmica ser baseado no número de artigos publicados podem também estar relacionado ao grande número de RS publicadas ${ }^{22}$.
Algumas limitações do estudo devem ser pontuadas. A busca foi restrita a apenas uma base de dados o que pode acarretar algum tipo de viés. Além disso, os achados são baseados no que é reportado nos estudos e é possível que algumas RS tenham sido conduzidas de forma mais rigorosa do que foi reportado e vice-versa.

\section{Conclusão}

Muitas revisões sobre a longevidade de restaurações vêm sendo publicadas na literatura odontológica sendo conduzidas e reportadas de maneira diferente o que pode impactar diretamente na qualidade do estudo e na aplicabilidade dos resultados. Estratégias devem ser incentivadas para que as revisões sistemáticas sejam melhor utilizadas como método de pesquisa e consequentemente os resultados reflitam em melhor atendimento por profissionais da saúde e melhora na saúde de pacientes.

\section{Agradecimentos}

Agradecemos à Biblioteca da Faculdade de Odontologia da Universidade Federal de Pelotas pelo fornecimento de alguns artigos para o desenvolvimento do estudo. Esse estudo é resultado da disciplina Metodologia do Aprendizado e Pesquisa II do curso de graduação da Faculdade de Odontologia da Universidade Federal de Pelotas.

\section{Abstract}

Several reviews evaluating the longevity of composite resin restorations in posterior teeth have been published and it is important that these reviews are better understood. Objective: this study aimed to investigate the conduction and reporting characteristics of systematic reviews that evaluated the longevity of composite resin restorations in posterior teeth. Materials and method: a search was conducted in MedLine (via Pubmed) to identify reviews analyzing only the longevity of composite resin restorations and reviews comparing composite resin restorations to other materials in posterior teeth. The studies were selected independently by two reviewers, initially based on the reading of titles/abstracts and later based on the reading of the full text. The reporting and conduction characteristics of the journals that published the studies were extracted. Tables were created to summarize data. Results: a total of 11 articles were included in the study through the qualitative synthesis. Most of the reviews performed meta-analysis (63.6\%) and mentioned the terms "systematic review/ meta-analysis" in the title or abstract (90.9\%). Seven reviews $(63.6 \%)$ did not mention the use of any type of reporting guideline. A great portion of the reviews $(54.5 \%)$ did not include only randomized clinical trials, and eight studies (72.2\%) evaluated the risk of bias and presented a flowchart for study selection. Conclusions: it may be concluded that several reviews on the longevity of restorations have been published in the dental 
literature, and many of them are conducted and reported differently, which may directly affect the quality of studies and the applicability of results.

Keywords: Longevity. Permanent dental restoration. Composite resins. Review.

\section{Referências}

1. Demarco FF, Corrêa MB, Cenci MS, Moraes RR, Opdam NJ. Longevity of posterior composite restorations: not only a matter of materials. Dent Mater 2012; 28(1):87-101.

2. Lynch CD, Opdam NJ, Hickel R, Brunton PA, Gurgan S, Kakaboura A, et al. Guidance on posterior resin composites: academy of operative dentistry - European section. J Dent 2014; 42(4):377-83.

3. Van de Sande FH, Collares K, Correa MB, Cenci MS, Demarco FF, Opdam NJM. Restoration survival: revisiting patients' risk factors through a systematic literature review. Oper Dent 2016; 41(S7):S7-S26.

4. Liberati A, Altman DG, Tetzlaff, J, Mulrow C, Gøtzsche PC, Ioannidis J P, et al. The PRISMA statement for reporting systematic reviews and meta-analyses of studies that evaluate health care interventions: explanation and elaboration. Ann Intern Med 2009; 151(4):W-65.

5. Page MJ, Shamseer L, Altman DG, Tetzlaff J, Sampson M, Tricco AC, et al. Epidemiology and Reporting Characteristics of Systematic Reviews of Biomedical Research: A CrossSectional Study. PLoS Med 2016; 13(5):e1002028.

6. Moher D, Shamseer L, Clarke M, Ghersi D, Liberati A, Petticrew M, et al. Preferred reporting items for systematic review and meta-analysis protocols (PRISMA-P) 2015 statement. Syst Rev 2015; 4(1):1.

7. U.S. National Libray of Medicine [acesso em 13 ago 2016]. Disponível em: https://www.nlm.nih.gov/.

8. Angeletaki F, Gkogkos A, Papazoglou E, Kloukos D. Direct versus indirect inlay/onlay composite restorations in posterior teeth. A systematic review and meta-analysis. J Dent 2016 ; 53:12-21.

9. Antony K, Genser D, Hiebinger C, Windisch F. Longevity of dental amalgam in comparison to composite materials. GMS Health Technol Assess 2008; 13(4):12.

10. Ástvaldsdóttir Á, Dagerhamn J, van Dijken JW, Naimi-Akbar A, Sandborgh-Englund G, Tranæus S, et al. Longevity of posterior resin composite restorations in adults-A systematic review. J Dent 2015; 43(8):934-54.

11. Chabouis HF, Faugeron VS, Attal JP. Clinical efficacy of composite versus ceramic inlays and onlays: a systematic review. Dental Materials 2013; 29(12), 1209-18.

12. Rasines Alcaraz MG, Veitz $\square$ Keenan A, Sahrmann P, Schmidlin PR, Davis D, Iheozor $\square$ Ejiofor Z. Direct composite resin fillings versus amalgam fillings for permanent or adult posterior teeth. Cochrane Database Syst Rev 2014;(3):CD005620.

13. Heintze SD, Rousson V. Clinical effectiveness of direct class II restorations-a meta-analysis. J Adhes Dent 2012; 14(5):407-31.

14. Mickenautsch S, Yengopal V. Failure rate of direct highviscosity glass-ionomer versus hybrid resin composite restorations in posterior permanent teeth- a systematic review. Open Dent J 2015; 22(9):438-48.

15. Moraschini V, Fai CK, Alto RM, dos Santos GO. Amalgam and resin composite longevity of posterior restorations: a systematic review and meta-analysis. J Dent 2015; 43(9):1043-50.
16. Morimoto S, de Sampaio FR, Braga MM, Sesma N, Özcan M. Survival rate of resin and ceramic inlays, onlays, and overlays A systematic review and meta-analysis. J Dent Res 2016; 95(9):985-94.

17. Opdam NJM, Van de Sande FH, Bronkhorst E, Cenci MS, Bottenberg P, Pallesen U, et al. Longevity of posterior composite restorations A systematic review and meta-analysis. J Dent Res 2014; 93(10):943-9.

18. Grivas E, Roudsari RV, Satterthwaite JD. Composite inlays: a systematic review. Eur J Prosthodont Restor Dent 2014; 22(3):117-24.

19. Page MJ, Shamseer L, Altman DG, Tetzlaff J, Sampson M, Tricco AC, et al. Epidemiology and Reporting Characteristics of Systematic Reviews of Biomedical Research: A Cross-Sectional Study. PLoS Med 2016; 13(5):e1002028.

20. Page MJ, Moher D. Mass Production of Systematic Reviews and Meta-analyses: An Exercise in Mega-silliness? Milbank Q 2016; 94(3):515-9.

21. Ioannidis J. The Mass Production of Redundant, Misleading, and Conflicted Systematic Reviews and Meta-analyses. Milbank Q 2016; 94(3):485-514.

22. Moher D, Glasziou P, Chalmers I, Nasser M, Bossuyt PM, Korevaar DA, et al. Increasing value and reducing waste in biomedical research: who's listening? Lancet 2016; 387(10027):1573-86.

Endereço para correspondência:

Rafael Sarkis-Onofre

Rua Gonçalves Chaves, 457/502

96015-560 Pelotas, RS, Brasil

E-mail: rafaelonofre@gmail.com

Recebido: 29/11/2016. Aceito: 19/03/2017. 